DOE/PC/91297--T1

DE92 012553

DOE/PC/91297-F /

1992

Quarterly Technical Progress Report

(December 1, 1991 to February 28, 1992)

\title{
A COMPUTATIONAL MODEL FOR \\ COAL TRANSPORT AND COMBUSTION \\ Grant Number：DE-FG22-91PC91297
}

\begin{abstract}
Goodarz Ahmadi
Department of Mechanical and Aeronautical Engineering

Clarkson University
\end{abstract}

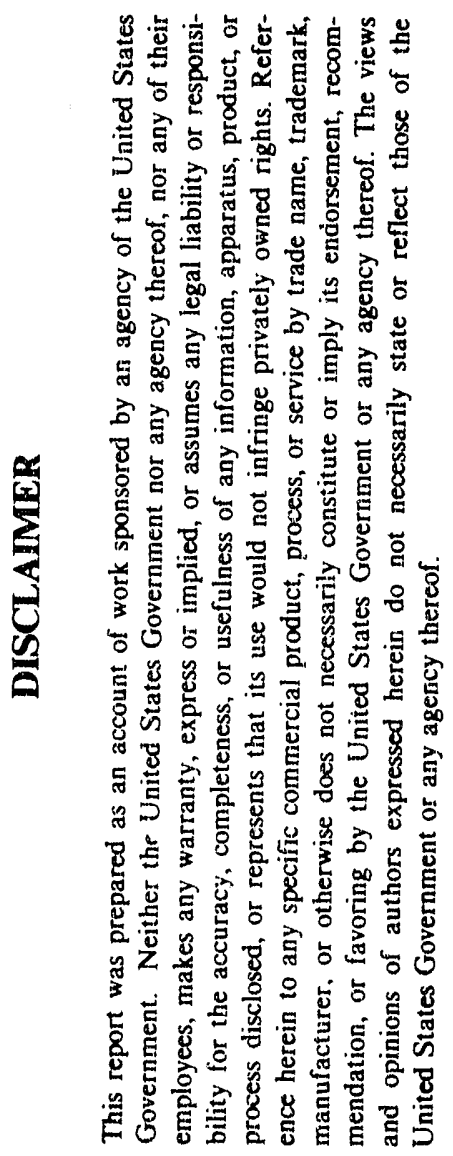

Submitted to

U.S. Department of Energy

Pittsburgh Energy Technology Center

\author{
Attention: \\ Dr. Mehrdad Massoudi \\ Project Officer \\ How
}

DISTRIBUTION OF THIS DOCIJMENT IS UNLIMITEO 


\section{A COMPU'TATIONAL MODEL FOR}

\section{COAL TRANSPORT AND COMBUSTION}

Grant Number: DE-FG22-91PC91297

Project Period: September 1, 1991 to August 31, 1994

Contract Recipient: Clarkson University

Project Principal Investigator: Goodarz Ahmadi

DOE Project Officer: Dr. Mehrdad Massoudi

\section{SUMMARY}

In the period of December 1, 1991 to February 28, 1992 further progress has been made in formulation of the thermodynamically consistent rate dependent model for turbulent two-phase flows. The thermodynamically admissible constitutive equations were derived, and the case of a simple shear flow was also studied.

A stress transport model for rapid granular flows in a rotating frame of reference was formulated. The thermodynamically consistent constitutive equations were derived. It was shown that the model contains the existing kinetic theories as special limiting cases. The model predictions for the special case of rapid simple shear flows were compared with the available simulation data.

The kinetic model for rapid flows of granular materials, which includes frictional losses, was used for studing several parallel shear flows. These include Couette and gravity flows.

Progress has been made in developing a computational model for rapid granular flows in complex geometries. The design for the experimetal simple shear flow device was also improved, and the construction of the device was initiated. 


\section{PROGRESS REPORT}

\section{A THERMODYNAMICALLY CONSISTENT RATE-DEPENDENT MODEL FOR TURBLLENT TWO-PHASE FLOWS}

Based on the averaged Clausius-Duhem inequality for multiphase mixtures in a turbulent state of motion, thermodynamically admissible expressions for the phasic stress tensors, heat and fluctuation energy flux vectors were derived. The developed stress constitutive equations were rate-dependent and nonlinear. For dense two-phase solid particles-liquid mixtures, the material parameters of the model were evaluated from the limiting conditions of rapid flows of dry spherical granular particles, and single-phase turbulent fluid flow. The special case of simple shear flows of glass beads-water mixtures were studied, and results concerning phasic stresse: and normal stress differences were obtained.

Figures 1 and 2 show variations of nondimensional first and second normal stress differences with solid volume fraction for different values of restitution coefficient. It is observed that the first normal stress difference is much larger than the second one and the anisotropy in normal stress components decreases as solid volume fraction increases. Furthermore, variaticns of restitution coefficient do not have a significant effect on first and second normal stress differences for solid-liquid mixtures. Additional results (not shown here) indicate that for the relatively dense two-phase mixtures, the rnain contributions to the normal stress differences are made by the fluid phase. At present, experimental confirmation of these results is not possible due to the lack of data for normal stress differences in dense two-phase mixtures. Verification of this result is part of the experimental phase of this project. 


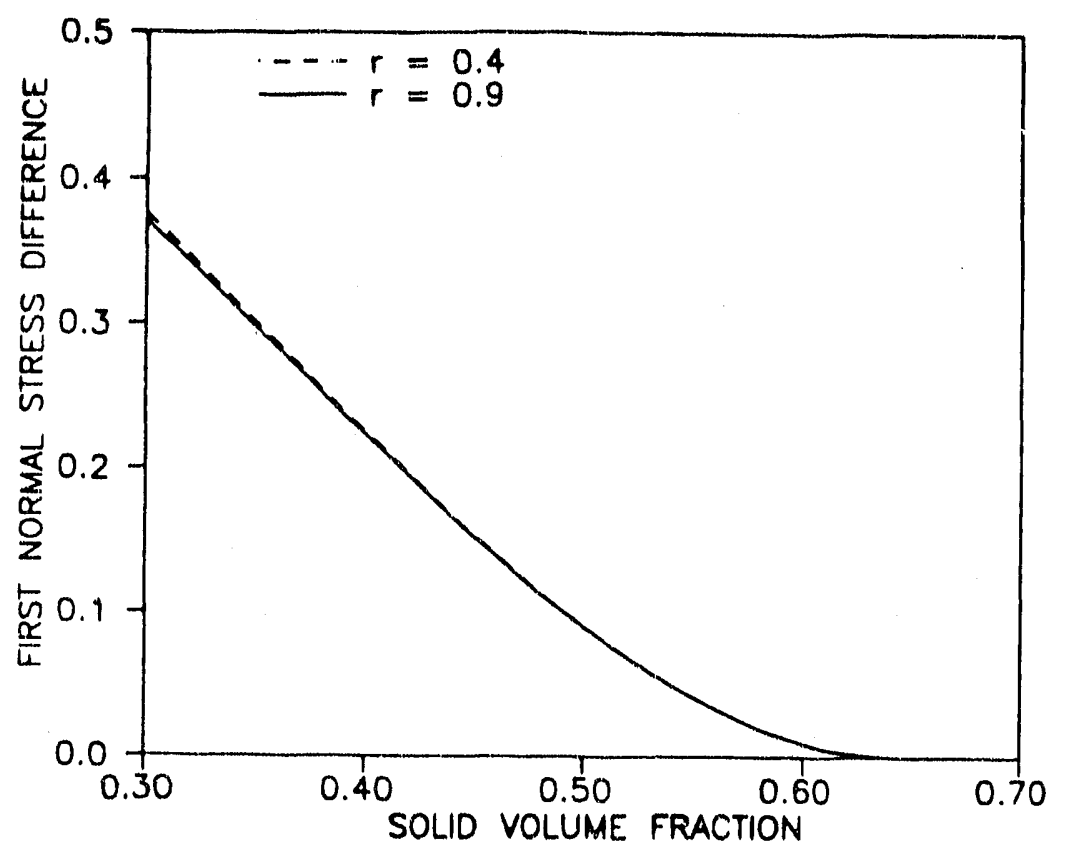

Figure 1. Variations of nondimensional first normal stress difference with solid volume fraction for different restitution coefficients.

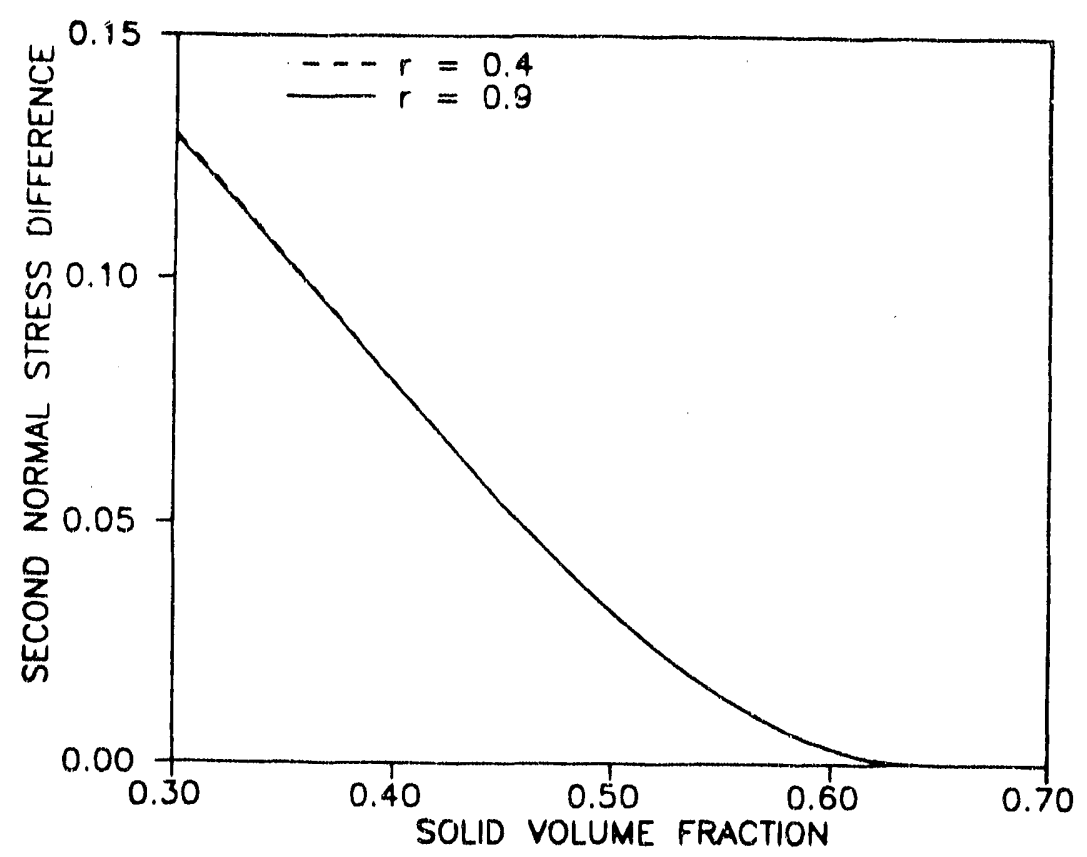

Figure 2. Variations of nondimensional second nomal stress difference with solid volume fraction for different restitution coefficients. 
For two-phase flows, the density ratio, $S_{0}$, plays a significant role in the overall behavior of the mixture. Figures 3 and 4 show variations of first and second normal stress differences with solid volume fraction for different values of density ratio. The stresses are nondimensionalized by division to the mixture density, square of shear rate and square of particll diameter. Here, a coefficient of restitution of $r=0.55$ and a particle Reynolds number of 200 for different density ratios are used. It is observed that as the density ratio increases, the nondimensional normal stress differences decrease. The effect of density ratio is more pronounced for low values of solid volume fraction. As noted before, the normal stress differences in two-phase mixture are mainly due to fluid phase. In particular, the second normal stress differences shown in Figure 4 are almost entirely contributed by the fluid phase. As the density ratio increases, the mixture density increases and the nondimensional normal stress diferences decrease.

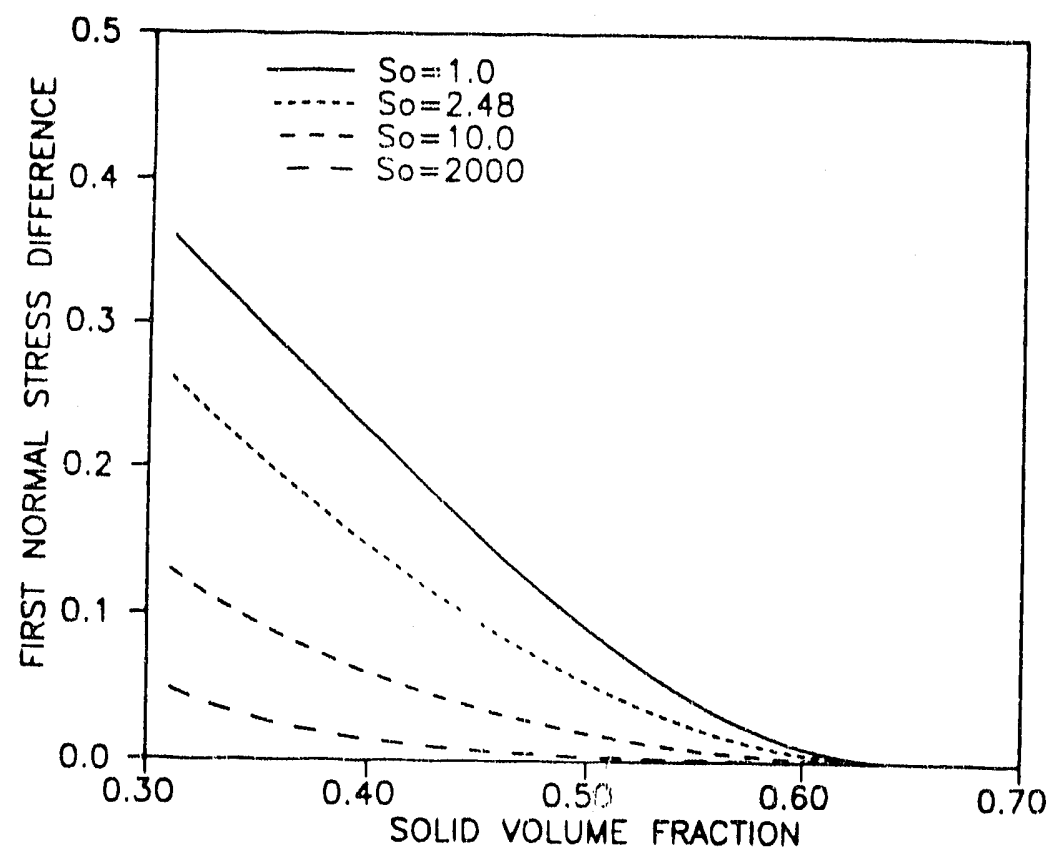

Figure 3. Variations of nondimensional firs: normal stress difference with solid volume fraction for different density ratios. 


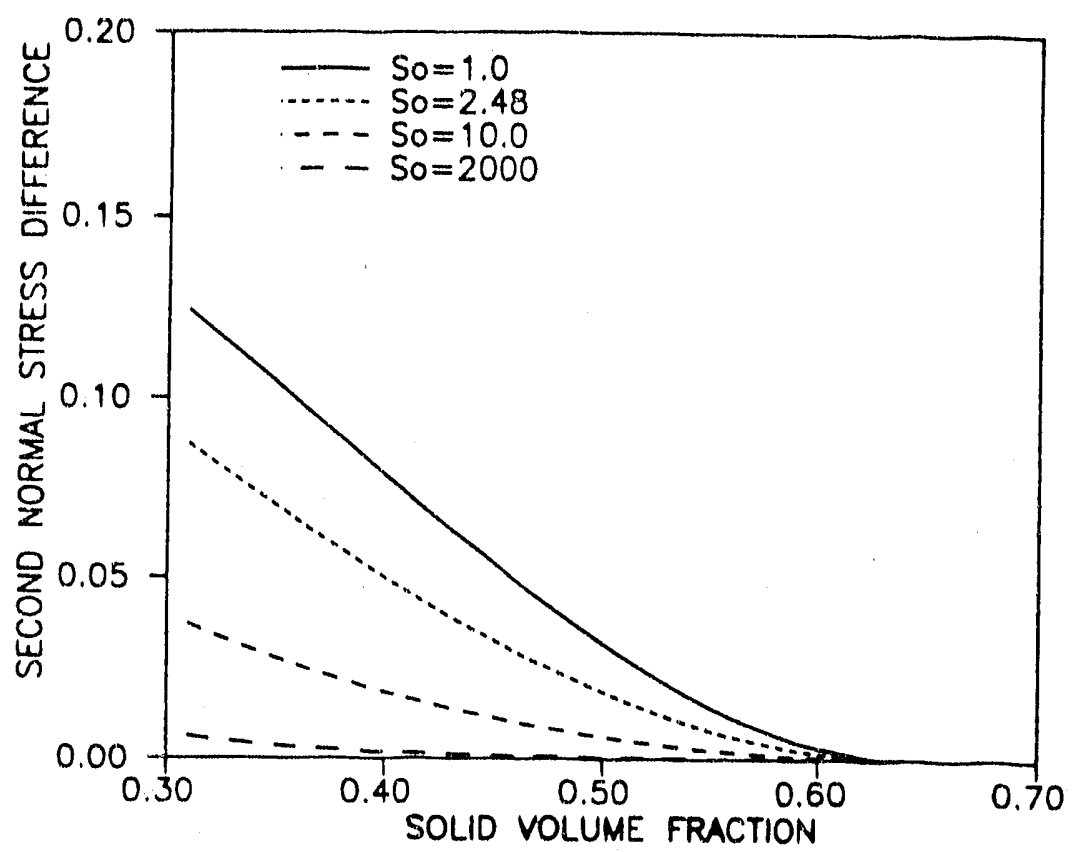

Figure 4. Variations of nondimensional second normal stress difference with solid volume fraction for different density ratios.

\section{A THERMODYNAMICALLY CONSISTENT STRESS TRANSPORT MODEL FOR RAPID GRANULAR FLOWS}

The basic conservation laws governing the flow of rapid granular materials in a rotating frame of reference along with a transport equation for the granular kinetic stress tensor and the corresponding Clausius-Duhem inequality were considered. The thermodynamic of the mean granular motion was studied and based on the averaged entropy inequality, constitutive equations for the pressure-strain, collisional stress, and flux of granular stress tensors were formulated. The model parameters for the special case of spherical nearly elastic particles were evaluated. The resulting stress transport model was used, and the case of rapid simple shear flow of a collection of particles was analyzed. The preliminary results for the granular stresses and the normal stress differences were compared with the available simulation data and good agreement was observed. 


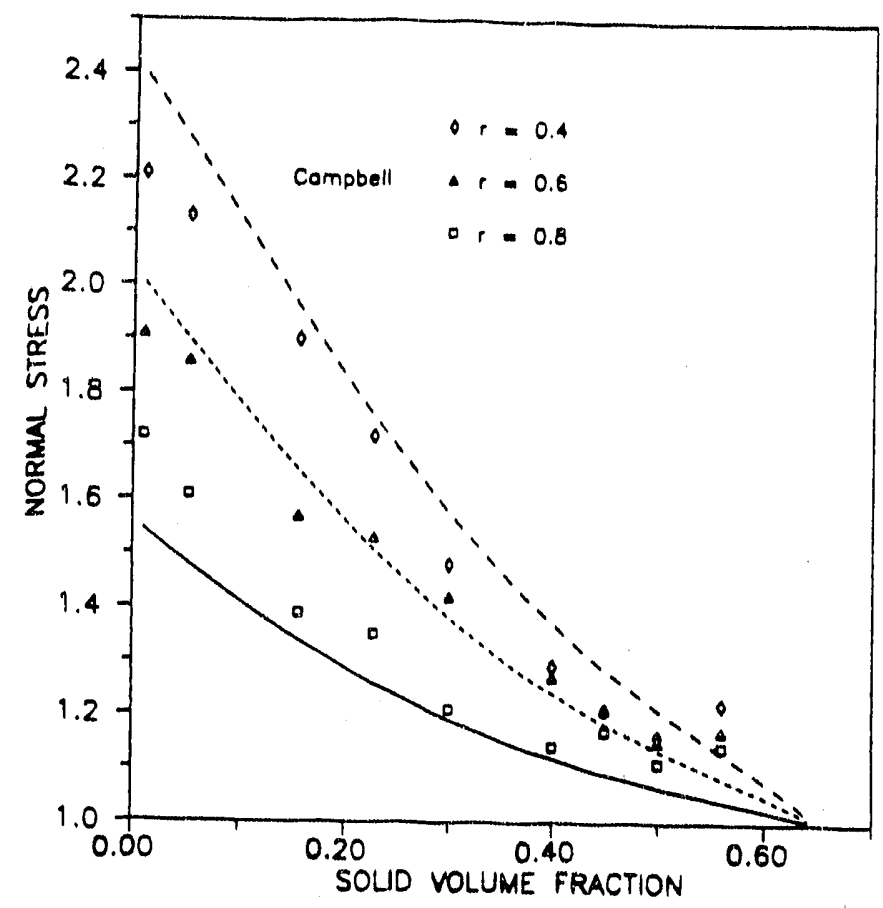

Figure 5. Variations of nondimensional first normal stress component with solid volume fraction.

The predicted nondimensional normal stresses versus solid volume fraction are shown in Figures 5-7 for restitution coefficients of $0.4,0.6$ and 0.8 . The corresponding simulation results of Campbell (1988) for the rough (frictional) spheres are also reproduced in these figures for comparison. It is observed that the model predictions for the first normal stress component are in good agreement with the simulation data. There are, however, certain deviations between the predicted second and third normal stress components and the simulation data. As noted before, the parameters of the model are evaluated for frictionless nonrotating spherical particles, while the simulation results are for frictional particles and includes particle's rotational effects. Thus, the observed qualitative agreement is quite encouraging. 


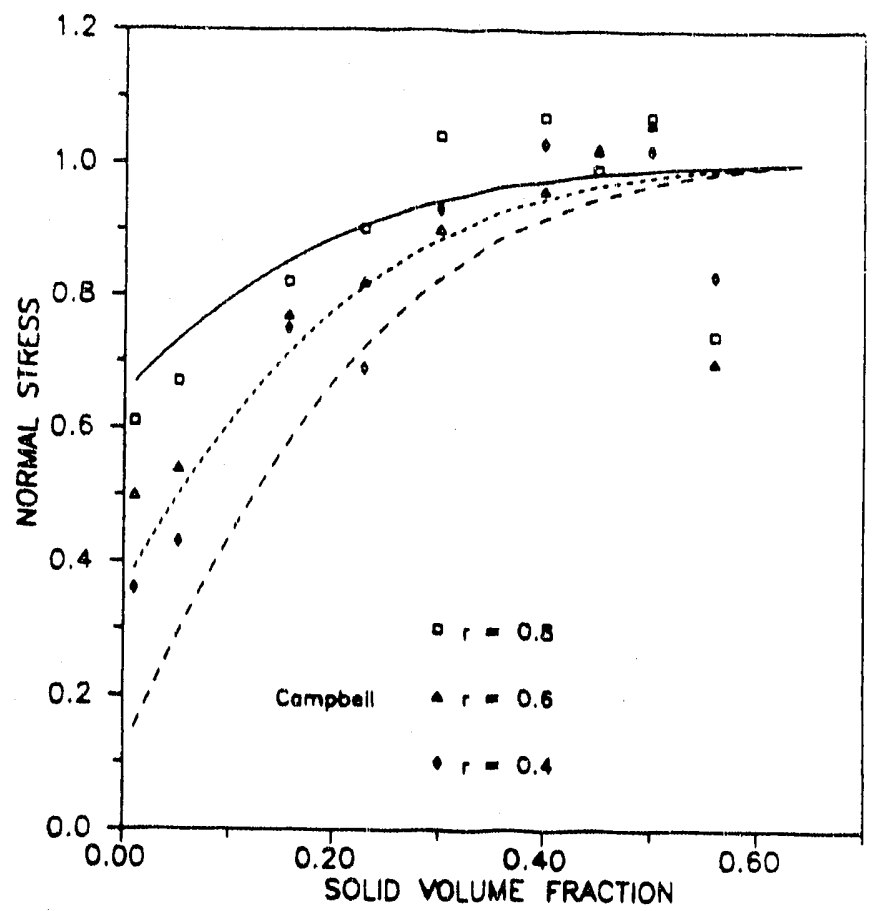

Figure 6. Variations of nondimensional second normal stress component with solid volume fraction.

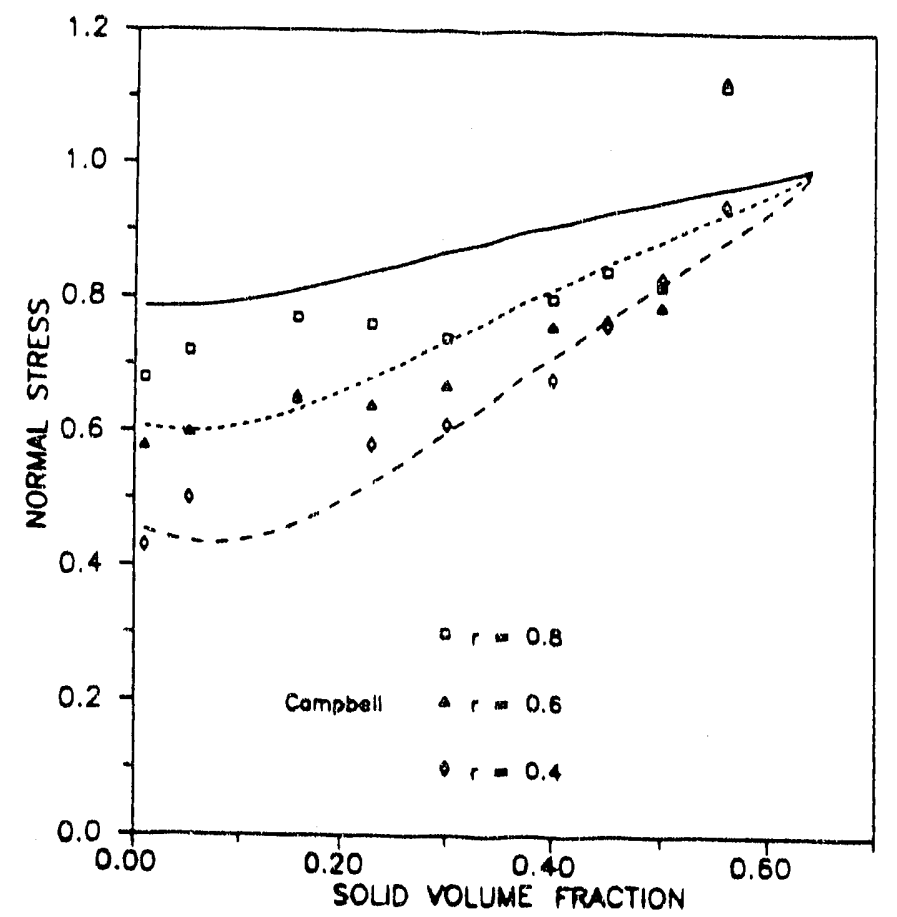

Figure 7. Variations of nondimensional third normal stress component with solid volume fraction. 


\section{ANALYSIS OF RAPID GRANULAR FLOWS INCLUDING FRICTIONAL LOSSES}

Rapid granular parallel flows were considered. The previously developed kinetic model for dry particulate flows which includes frictional losses was used in the analysis. The equations of motion were solved using a fourth-order Runge-Kutta numerical scheme. The mean velocity, the fluctuation kinetic energy and the solid volume fraction profiles were evaluated under a variety of conditions. Particular attention was given to the effects of friction coefficient. The results for the granular Couette flows are summarized in this section. Figures 8 and 9 display typical velocity, solid volume fraction and fluctuation kinetic energy profiles across the flow for zero coefficient of friction and different boundary conditions. A coefficient of restitution of $r=0.9$ for particles was also used. These figures show that the mean velocity varies almost linearly across the channel. For different values of energy loss coefficient at the upper plate, the solid volume fraction and fluctuation kinetic energy have different profiles.

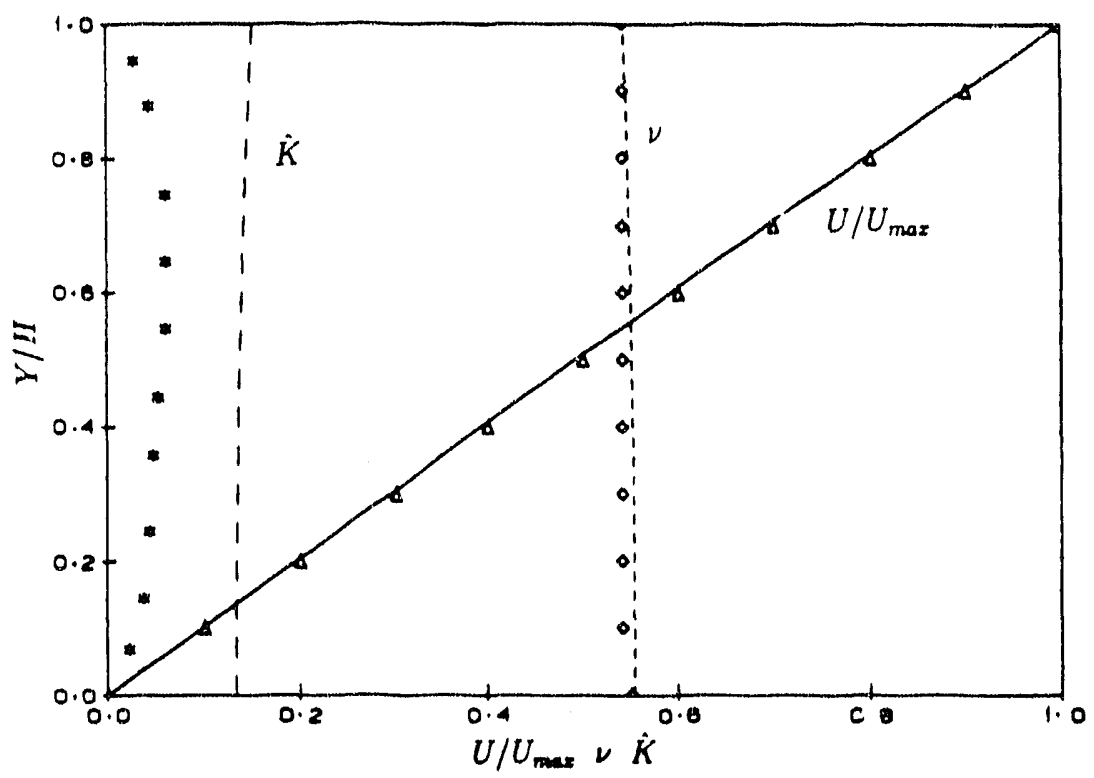

Figure 8. Variations of nondimensional velocity, solid volume fraction and fluctuation kinetic energy profiles. Comparison with the simulations of Campbell and Brennen (1985) 
Figure 8 also shows a comparison between the results of the present model and the computer simulations of Campell and Brennen (1985) for two-dimensional circular disks. It is observed that the predictions of the present model are, generally, in good agreement with the simulation data. However, the predicted fluctuation energy profile somewhat overestimates the simulation results.

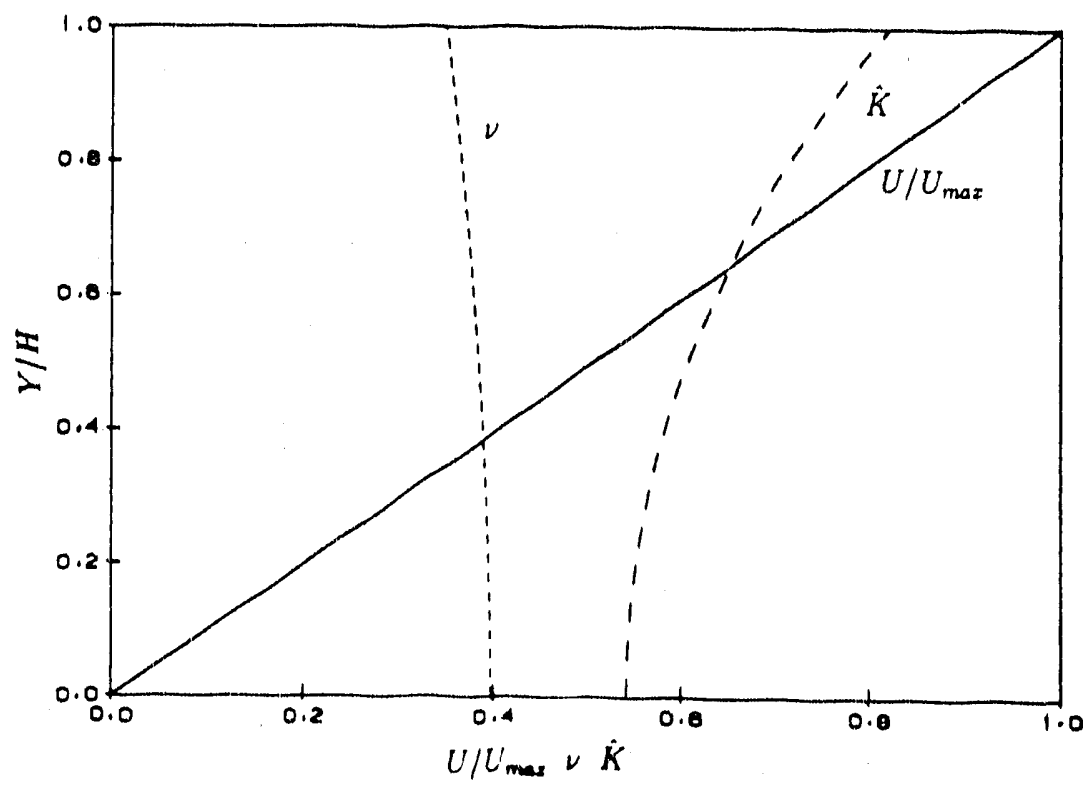

Figure 9. Variations of nondimensional velocity, solid volume fraction and fluctuation kinetic energy profiles.

A series of numerical experiments for different values of friction coefficient are also carried out and the corresponding solid volume fraction and fluctuation kinetic energy profiles ar. presented in figures 10 and 11, respectively. The values of solid volume fraction at the centerline and fluctuation kinetic energy at the boundaries are kept fixed in these analyses. It is observed that the solid volume fraction and fluctuation kinetic energy decrease as friction coefficient increases. The nondimensional mean velocity profiles which do not change appreciably with friction and remain almost linear are not shown here. 


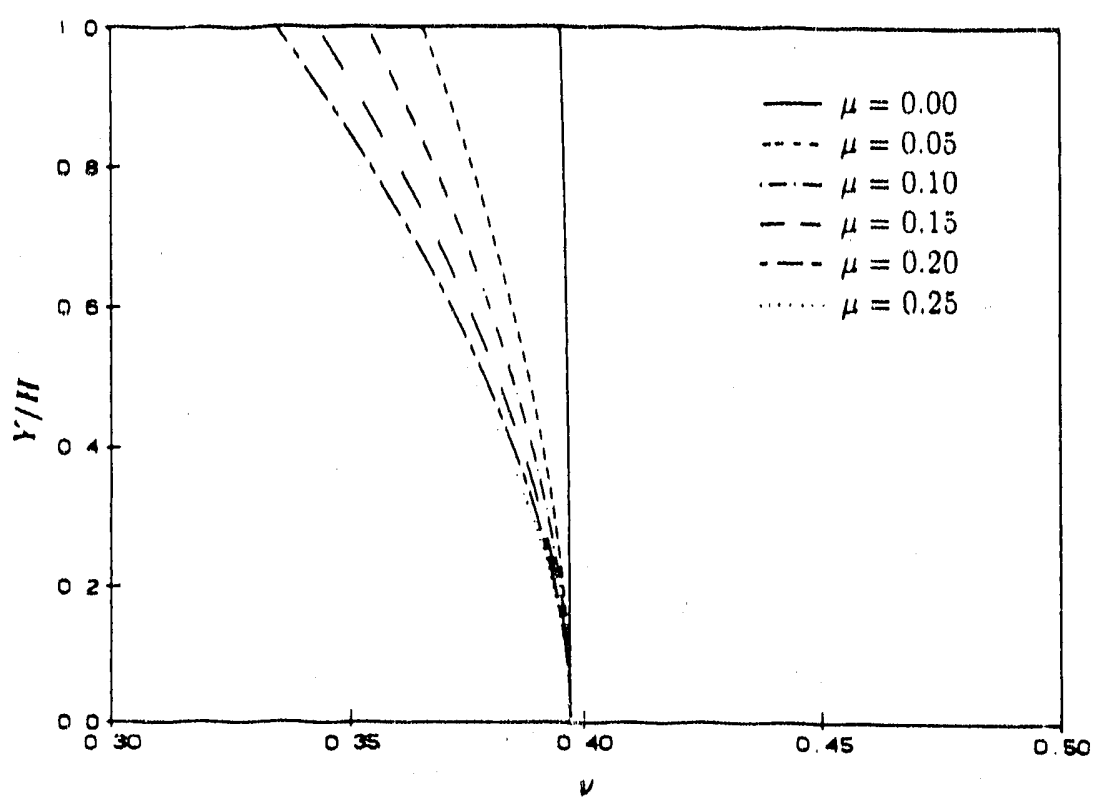

Figure 10. Variations of solid volume fraction profiles for different friction coefficients.

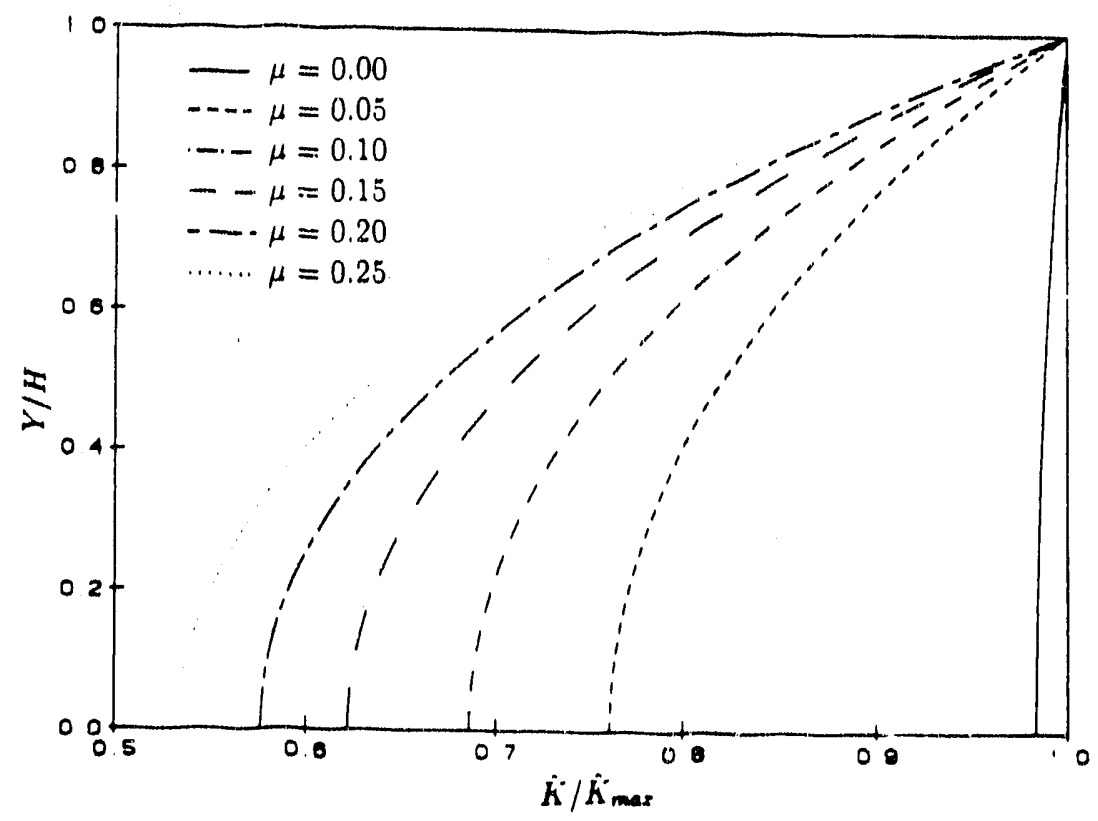

Figure 11. Variations of nondimensional fluctuation kinetic energy profiles for different friction coefficients. 


\section{COMPUTATIONAL MODEL DEVELOPMENT}

Formulation of appropriate computational scheme of solution is being studied. It is recognized that for developing an appropriate two-fluid computational code the starting single phase code need to be compressible. A compressible flow code is being studied for extension to two-phase flows solid-liquid or gas flows. In andition, the discrete element procedure is being used for analyzing rapid granular flows down a chute.

\section{EXPERIMENTAL STUDY}

The main objective of the experimental effort is to verify the foundation of the thermodynamical formulation. The other main goals are to provide reliable data for velocity, concentration and fluctuation energy profiles for a simple shear flow of granular materials and/or turbulent two-phase flows in order to verify the theoretical predictions. Several designs for the simple shear flow device are considered. The most suitable design for the granular shear flow device was selected. Construction of the device was initiated. 

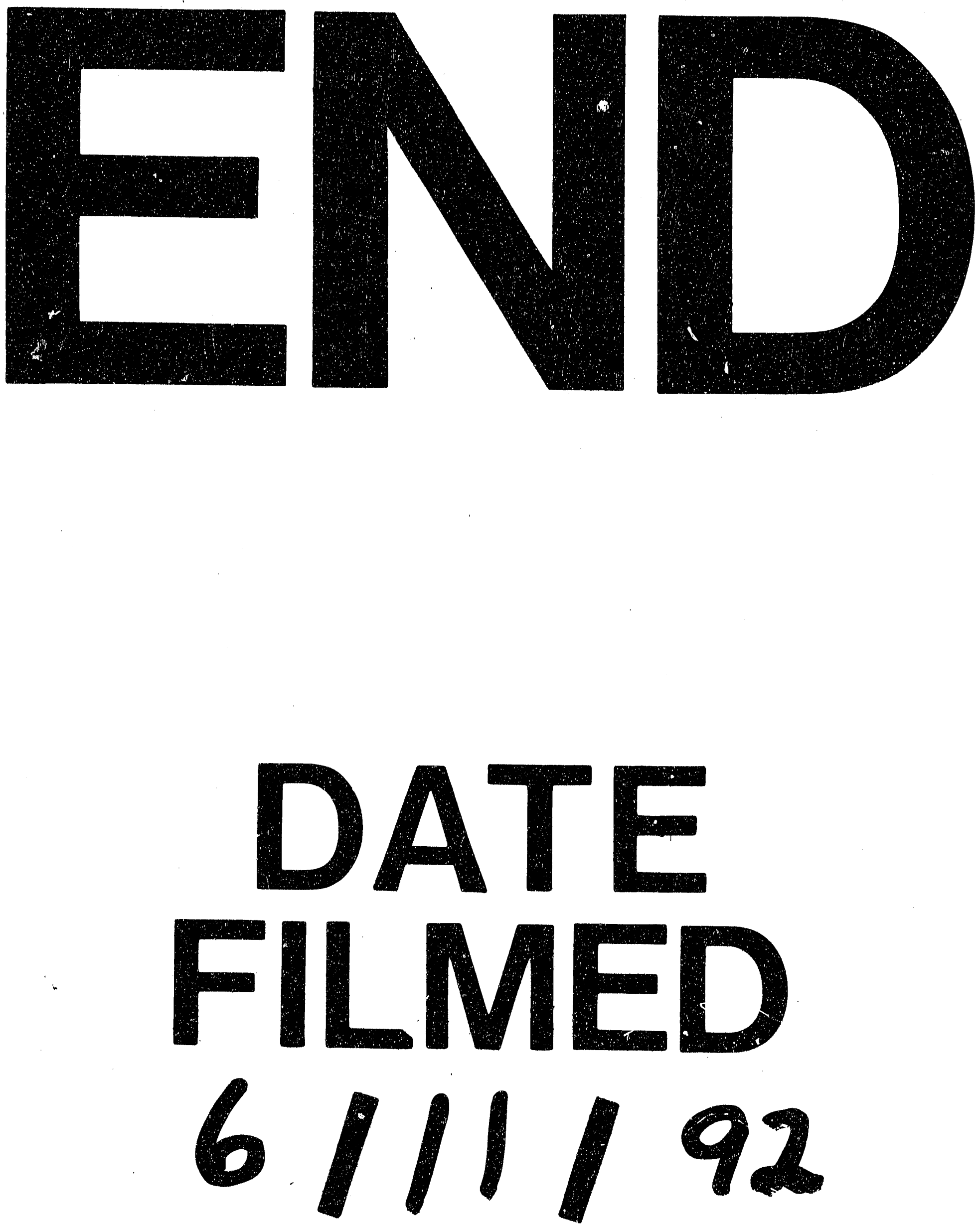


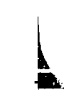

